03,04

\title{
Отклик на однородное электрическое поле в расчетах электронной структуры кристаллов с базисом локализованных орбиталей
}

\author{
() А.Б. Гордиенко, Д.И. Филиппов ๘ \\ Кемеровский государственный университет, \\ Кемерово, Россия \\ ๑ E-mail: filippovdaniil@kemsu.ru \\ Поступила в Редакцию 5 декабря 2018 г. \\ В окончательной редакции 15 января 2019 г. \\ Принята к публикации 15 января 2019 г.
}

\begin{abstract}
Рассматривается вычисление отклика на внешнее, однородное электрическое поле в расчетах электронной структуры кристаллов с использованием базиса локализованных функций в форме псевдоатомных орбиталей. Получены основные уравнения, учитывающие поправки на неполноту и неортогональность базиса, включая расчетные формулы для диэлектрического тензора и динамических зарядов. Результаты реализации предложенного метода вычислений обсуждаются на примере расчетов, выполненных для соединений различного типа.
\end{abstract}

DOI: 10.21883/FTT.2019.06.47680.333

\section{1. Введение}

Теория функционала плотности (DFT) $[1,2]$ в сочетании с теорией псевдопотенциала [3] в настоящее время является одним из наиболее эффективных подходов для теоретического исследования и описания свойств кристаллических твердых тел. В таком варианте, благодаря гладкому характеру одночастичных функций в области ионного остова, для решения уравнений Кона-Шэма [2] возможно использование базиса плоских волн (PW), функции которого образуют полную, ортогональную систему и по форме определяются только типом кристаллической решетки. Указанные свойства базиса PW определяют его преимущества по сравнению с другими типами базисов, что, в частности, относится к простоте реализации вычислений, поскольку при этом полностью сохраняется структура формально точных уравнений как для стандартного способа решения одночастичных уравнений [3], так и прямой минимизации полной энергии [4], а также контролю точности, которая во всех случаях определяется только размерностью базиса. При этом преимущества базиса PW характерны не только для расчета основного состояния, но сохраняются также при вычислении отклика системы на изменение ее внешней конфигурации (структура, атомный состав, силовые поля). Основные принципы решения этой задачи в рамках теории линейного отклика сформулированы в $[5,6]$, а также в $[7,8]$, где, в том числе, рассмотрены и практические аспекты реализации расчетов с PW-базисом, составляющие основу достаточно популярных программных пакетов abinit [9] и QuantumEspresso [10].

Несмотря на отмеченные выше важные преимущества, PW-базис имеет и ряд существенных недостатков. Прежде всего, в этом случае полноэлектронные расчеты для реальных систем, представляющих интерес, становятся практически невозможными из-за исключитель- но больших размерностей такого базиса. Аналогичные трудности могут возникать и в псевдопотенциальных расчетах, например для систем с переходными элементами, атомы которых содержат достаточно локализованные состояния, которые, в силу заметного перекрывания с состояниями внешних электронов, не могут быть отнесены к ионному остову однозначно и без заметных потерь качества получаемого псевдопотенциала, [11]. Решением этих и других проблем является переход к методам расчета, использующим базис, который по способу построения учитывает характер атомных состояний: ортогонализованные плоские волны (OPW), присоединенные плоские волны (APW, LAPW, FP-LAPW), muffin-tin орбитали (LMTO, FP-LMTO), линейные комбинации атомных орбиталей (LCAO), смешанный базис $(\mathrm{LCAO}+\mathrm{PW})$. Общим свойством для всех приведенных базисов, помимо неполноты и неортогональности, является зависимость от положения атомов, что, в совокупности, приводит к существенной модификации и усложнению методов расчета как основного состояния, так и его отклика. Последовательное вычисление слагаемых ряда теории возмущений

$$
E(\lambda)=E^{(0)}+\lambda E^{(\lambda)}+\lambda^{2} E^{(\lambda \lambda)}+\ldots,
$$

где $E-$ энергия основного состояния, $\lambda-$ внешний параметр, уже в первом порядке (силы, напряжения и т.д.) приводит к дополнительным вкладам (орбитальные силы Пулэя) [12] в формуле Геллмана-Фейнмана [13,14], которые, как следствие „2n+1“ теоремы Вагнера (см. напр. $[7,8])$, не требуют вычисления поправок волновых или одночастичных функций выше нулевого порядка. Поправка первого порядка в разложении

$$
\psi(\lambda)=\psi^{(0)}+\lambda \psi^{(\lambda)}+\ldots
$$

является необходимой для определения величины $E^{(2)}$, которая, в зависимости от типа возмущения, является 
основной для расчета колебательных спектров и диэлектрических свойств твердых тел [6]. Методы вычисления функций $\psi^{(\lambda)}$ как решений самосогласованного уравнения Штернхаймера для базиса $\mathrm{PW}$ рассматривались в $[5-8,15]$, а для базиса локализованных функций в [16-20]. Несмотря на различия в деталях, все отмеченные методы являются эквивалентными, представляя собой, так или иначе, варианты решения (обращения) матричного уравнения Штернхаймера, которое первоначально рассматривалось в рамках метода Хартри-Фока в [21] для молекулярных систем. Интересно отметить, что непосредственно этот подход в расчетах для периодических систем практически не используется и, пожалуй, единственным примером является работа [20], где вычисления в рамках теории линейного отклика с орбитальным базисом реализованы в координатном пространстве. Кроме того, вычисление отклика на внешнее, однородное электрическое поле детально рассматривается только в работах $[6-8,15]$ для базиса $\mathrm{PW}$, а в остальных либо ограничено достаточно краткими комментариями, либо не приводится вообще, хотя является важной составной частью расчета тензоров эффективных, динамических зарядов и диэлектрической проницаемости.

Целью настоящей работы является реализация вычислений функций отклика с использованием базиса локализованных функций в форме псевдоатомных орбиталей [22] на основе подхода [21], а также оценка точности вычислений на примере соединений различного типа.

\section{2. Вычисление функций отклика с базисом локализованных функций}

В рамках теории функционала плотности $[1,2]$ одночастичные функции и соответствующий спектр энергий определяются из решения уравнений Кона-Шэма [2]

$$
\hat{H} \psi_{n}=E_{n} \psi_{n},
$$

где $\hat{H}$ - гамильтониан системы

$$
\hat{H}=-\frac{1}{2} \nabla^{2}+\hat{V}_{\mathrm{i}-\mathrm{e}}+\hat{V}_{\mathrm{Hxc}[\rho]},
$$

и, соответственно, $\hat{V}_{\mathrm{i}-\mathrm{e}}-$ потенциал взаимодействия ионов (в приближении псевдопотенциала) и электронов, $\hat{V}_{\mathrm{Hxc}[\rho]}=\hat{V}_{\mathrm{H}[\rho]}+\hat{V}_{\mathrm{xc}[\rho]}-$ электростатический и обменнокорреляционный потенциалы, зависящие от электронной плотности

$$
\rho=\sum_{n} f_{n}|\psi|^{2},
$$

где $f_{n}$ - числа заполнения одночастичных состояний.

Представление решений (3) в виде разложения по базису функций $\varphi_{i}\left(i=1, N_{b}\right)$

$$
\psi_{n}=\sum_{i} C_{i n} \varphi_{i}
$$

позволяет преобразовать уравнения (3) к обобщенной алгебраической задаче на собственные значения и векторы, имеющей размерность $N_{b} \times N_{b}$

$$
\sum_{j}\left[H_{i j}-E_{n} S_{i j}\right] C_{j n}=0,
$$

с условием ортогональности

$$
\sum_{i, j} C_{i m}^{*} S_{i j} C_{j n}=\delta_{m n}
$$

где $H_{i j}$ и $S_{i j}$ матрицы гамильтониана и перекрывания

$$
H_{i j}=\left\langle\varphi_{i}|\hat{H}| \varphi_{j}\right\rangle, \quad S_{i j}=\left\langle\varphi_{i} \mid \varphi_{j}\right\rangle .
$$

Пусть гамильтониан (4) имеет вид

$$
\hat{H}=\hat{H}^{(0)}+\lambda \hat{H}^{(\lambda)}+\ldots
$$

В этом случае все величины в (7) становятся функциями параметра $\lambda$, и с точки зрения вычисления отклика одночастичных функций

$$
\psi_{n}^{(\lambda)}=\sum_{i}\left[C_{i n} \varphi_{i}^{(\lambda)}+C_{i n}^{(\lambda)} \varphi_{i}\right]
$$

основной является задача определения величин $C_{i n}^{(\lambda)}$, так как вычисление $\varphi^{(\lambda)}$ является тривиальным, поскольку для базисных функций зависимость от $\lambda$, как правило, известна в явной форме. Представляя все величины в уравнении (7) в виде, аналогичном (2), и ограничиваясь первым порядком по $\lambda$, получим уравнение

$$
\begin{aligned}
\sum_{j}\left[H_{i j}\right. & \left.-E_{n} S_{i j}\right] C_{j n}^{(\lambda)} \\
& =E_{n}^{(\lambda)} \sum_{j} S_{i j} C_{j n}-\sum_{j}\left[H_{i j}^{(\lambda)}-E_{n} S_{i j}^{(\lambda)}\right] C_{j n},
\end{aligned}
$$

которое является матричным аналогом уравнения Штернхаймера и его решение может быть найдено с помощью методов линейной алгебры. Как уже отмечено выше, в [21] предложен несколько иной способ решения уравнения (12), основанный на параметризации $C_{i n}(\lambda) \mathrm{c}$ помощью выражения

$$
C_{i n}(\lambda)=\sum_{m} U_{m n}(\lambda) C_{i m}
$$

с начальным условием, соответствующим невозмущенной задаче $(\lambda=0)$,

$$
U_{m n}(0)=\delta_{m n}, \quad C_{i n}(0)=C_{i n} .
$$

С учетом

$$
C_{i n}^{(\lambda)}=\sum_{m} U_{m n}^{(\lambda)} C_{i m}
$$

уравнение (12), после несложных преобразований, принимает вид

$$
\left[\mathscr{H}_{m n}^{(\lambda)}-E_{n} \mathcal{S}_{m n}^{(\lambda)}\right]+\left[E_{m}-E_{n}\right] U_{m n}^{(\lambda)}=E_{n}^{(\lambda)} \delta_{m n},
$$


где использованы обозначения

$$
\mathscr{H}_{m n}^{(\lambda)}=\sum_{i j} C_{i m}^{*} H_{i j}^{(\lambda)} C_{j n}, \quad \mathcal{S}_{m n}^{(\lambda)}=\sum_{i j} C_{i m}^{*} S_{i j}^{(\lambda)} C_{j n}
$$

Тогда из (16) для случая $m \neq n$ получим

$$
U_{m n}^{(\lambda)}=\frac{\mathscr{H}_{m n}^{(\lambda)}-E_{n} \mathcal{S}_{m n}^{(\lambda)}}{E_{n}-E_{m}}
$$

и для $m=n$

$$
E_{n}^{(\lambda)}=\mathscr{H}_{n n}^{(\lambda)}-E_{n} \mathcal{S}_{n n}^{(\lambda)},
$$

а диагональные значения $U_{n n}^{(\lambda)}$ можно определить из условия (8) в первом порядке по $\lambda$ как

$$
U_{n n}^{(\lambda)}=-\frac{1}{2} \mathcal{S}_{n n}^{(\lambda)}
$$

Заключительным шагом в случае общего типа возмущения $\lambda$ является вычисление отклика электронной плотности

$$
\rho^{(\lambda)}=2 \sum_{n} f_{n} \psi_{n}^{*} \psi_{n}^{(\lambda)},
$$

которое замыкает цикл самосогласованного решения уравнения Штернхаймера.

\section{3. Вычисление отклика на однородное электрическое поле для периодических систем}

Для обобщения приведенной выше схемы вычисления функций отклика на периодические системы необходимо учесть несколько особенностей. Прежде всего, в силу трансляционной симметрии одночастичные функции имеют вид

$$
\psi_{n \mathbf{k}}(\mathbf{r})=e^{i \mathbf{k r}} u_{n \mathbf{k}}(\mathbf{r}),
$$

где $u_{n \mathbf{k}}(\mathbf{r})$ - периодическая функция, $n-$ номер энергетической зоны, $\mathbf{k}$ - квазиимпульс и, таким образом, нумеруются с помощью комбинированного индекса $(n \mathbf{k})$; соответственно, разложение $\psi_{n \mathbf{k}}$ по базису блоховских функций $\varphi_{i k}$ запишется как

$$
\psi_{n \mathbf{k}}(\mathbf{r})=\sum_{i} C_{i n \mathbf{k}} \varphi_{i \mathbf{k}}(\mathbf{r})
$$

При наложении внешнего электрического поля напряженности $\mathscr{E}$ на кристалл, гамильтониан (10) принимает вид $^{1}$

$$
\hat{H}=\hat{H}^{(0)}+\sum_{\alpha=x, y, z} x_{\alpha} \mathscr{E}_{\alpha}
$$

и тогда величины, входящие в (17), с учетом независимости кинетической энергии, потенциала электрон-ионного

\footnotetext{
${ }^{1}$ В формуле (24) и далее используются атомные единицы: $e=m_{e}=\hbar=1, E_{n} \approx 27.211$.
}

взаимодействия, а также базисных функций в (6) и (23) от возмущения будут вычисляться как

$$
\mathscr{H}_{m n}^{\left(\mathscr{E}_{\alpha}\right)}=\sum_{i, j} C_{i m \mathbf{k}}^{*} V_{\mathrm{Hxc} . i j}^{\left(\mathscr{E}_{\alpha}\right)} C_{j n \mathbf{k}}+\left\langle u_{m \mathbf{k}}\left|x_{\alpha}\right| u_{n \mathbf{k}}\right\rangle, \quad \mathcal{S}_{m n}^{\left(\mathscr{E}_{\alpha}\right)}=0
$$

где

$$
\begin{gathered}
V_{\mathrm{Hxc} . i j}^{\left(\mathscr{E}_{\alpha}\right)}=\left\langle\varphi_{i \mathbf{k}}\left|V_{\mathrm{H}}^{\left(\mathscr{E}_{\alpha}\right)}+V_{\mathrm{xc}}^{\left(\mathscr{E}_{\alpha}\right)}\right| \varphi_{j \mathbf{k}}\right\rangle, \\
V_{\mathrm{H}}^{\left(\mathscr{E}_{\alpha}\right)}(\mathbf{r})=\int \frac{\rho^{\left(\mathscr{E}_{\alpha}\right)}\left(\mathbf{r}^{\prime}\right)}{\left|\mathbf{r}-\mathbf{r}^{\prime}\right|} d \mathbf{r}^{\prime}, \\
V_{\mathrm{xc}}^{\left(\mathscr{E}_{\alpha}\right)}(\mathbf{r})=\frac{\mathrm{d} V_{\mathrm{xc}}(\rho ; \mathbf{r})}{\mathrm{d} \rho} \rho^{\left(\mathscr{E}_{\alpha}\right)}(\mathbf{r})
\end{gathered}
$$

и отклик плотности ${ }^{2}$

$$
\rho^{\left(\mathscr{E}_{\alpha}\right)}(\mathbf{r})=\frac{2}{N_{\mathbf{k}}} \operatorname{Re} \sum_{n \mathbf{k}} f_{n \mathbf{k}} u_{n \mathbf{k}}^{*}(\mathbf{r}) u_{n \mathbf{k}}^{\left(\mathscr{E}_{\alpha}\right)}(\mathbf{r})
$$

Следует отметить, что вычисление дипольных матричных элементов $\left\langle u_{m \mathbf{k}}\left|x_{\alpha}\right| u_{n \mathbf{k}}\right\rangle$ в (25) в случае периодических систем требует специального рассмотрения, так как эти величины в данном случае являются плохо определенными. В [7] приведен детальный анализ этой проблемы и формально решение заключается в переходе к импульсному представлению, то есть

$$
\left\langle u_{m \mathbf{k}}\left|x_{\alpha}\right| u_{n \mathbf{k}}\right\rangle=i\left\langle u_{m \mathbf{k}} \mid u_{n \mathbf{k}}^{\left(k_{\alpha}\right)}\right\rangle,
$$

что требует предварительного вычисления функций $u_{n \mathbf{k}}^{\left(k_{\alpha}\right)}$, которые также могут быть определены с использованием общей схемы, приведенной выше и в данном случае требующей только одного шага (18).

\section{4. Тензоры диэлектрической проницаемости и эффективных зарядов}

Тензор диэлектрической проницаемости, как показано в [8], может быть вычислен как вторая производная полной энергии кристалла $\left(E_{\text {tot }}\right)$ по компонентам напряженности электрического поля

$$
\varepsilon_{\alpha \beta}^{\infty}=\delta_{\alpha \beta}-\frac{4 \pi}{\Omega_{0}} E_{\mathrm{tot}}^{\left(\mathscr{E}_{\alpha} \mathscr{E}_{\beta}\right)} .
$$

где $\Omega_{0}$ - объем элементарной ячейки кристалла. Первая производная определяет электронный вклад в поляризацию кристалла, и ее вычисление приводит к выражению

$$
P_{\alpha}^{(\mathrm{el})}=-\frac{i}{\Omega_{0} N_{\mathbf{k}}} \sum_{n \mathbf{k}} f_{n \mathbf{k}}\left\langle u_{n \mathbf{k}} \mid u_{n \mathbf{k}}^{\left(k_{\alpha}\right)}\right\rangle,
$$

после повторного дифференцирования которого можно получить, с учетом (31),

$$
\varepsilon_{\alpha \beta}^{\infty}=\delta_{\alpha \beta}-\frac{8 \pi i}{\Omega_{0} N_{\mathbf{k}}} \sum_{n \mathbf{k}} f_{n \mathbf{k}}\left\langle u_{n \mathbf{k}}^{\left(\mathscr{E}_{\alpha}\right)} \mid u_{n \mathbf{k}}^{\left(k_{\beta}\right)}\right\rangle .
$$

\footnotetext{
${ }^{2}$ Символ Re в (29) и аналогичных формулах далее может быть опущен в силу симметрии относительно обращения времени.
} 
Отметим, что выражение (33) совпадает с формулами, которые получаются при точном рассмотрении, а также для случая базиса PW [8], что в данном случае является простым следствием независимости базисных функций в (23) от возмущения в форме электрического поля и не приводит, таким образом, к дополнительным поправкам.

Динамические заряды имеют смысл коэффицента пропорциональности между изменением поляризациии на элементарную ячейку кристалла в направлении $\beta$ и смещением атома $a$ в направлении $\alpha$, либо между изменением силы действущей на атом $a$, в направлении $\alpha$, и изменением наложенного на кристалл внешнего однородного электрического поля в направлении $\beta$ :

$$
Z_{a, \alpha \beta}^{*}=\Omega_{0} \frac{\partial P_{\beta}}{\partial \tau_{a, \alpha}}=\frac{\partial F_{a, \alpha}}{\partial \mathscr{E}_{\beta}},
$$

где $\tau_{a}-$ положение атома $a$. Для настоящей работы более удобным является второе определение, так как оно позволяет вычислить эффективные заряды после определения отклика одночастичных функций на внешнее электрическое поле. Тогда, исходя из выражения для электронного вклада в силу, действующую на атом $a$

$$
\begin{aligned}
F_{a, \alpha}^{(\mathrm{el})}= & \frac{2}{N_{\mathbf{k}}} \sum_{n \mathbf{k}} f_{n \mathbf{k}} \sum_{i, j} C_{i n \mathbf{k}}^{*}\left\langle\varphi_{i \mathbf{k}}^{(a \alpha)}\left|\hat{H}-E_{n \mathbf{k}}\right| \varphi_{j \mathbf{k}}\right\rangle C_{j n \mathbf{k}} \\
& +\frac{1}{N_{\mathbf{k}}} \sum_{n \mathbf{k}} f_{n \mathbf{k}} \sum_{i, j} C_{i n \mathbf{k}}^{*}\left\langle\varphi_{i \mathbf{k}}\left|V_{\mathrm{i}-\mathrm{e}}^{(a \alpha)}\right| \varphi_{j \mathbf{k}}\right\rangle C_{j n \mathbf{k}}
\end{aligned}
$$

можно получить для экранирующей части эффективного заряда

$$
\begin{aligned}
& Z_{a, \alpha \beta}^{*(\mathrm{el})}=\frac{2}{N_{\mathbf{k}}} \sum_{n \mathbf{k}} f_{n \mathbf{k}} \sum_{i, j} C_{i n \mathbf{k}}^{*\left(\mathscr{E}_{\beta}\right)}\left[H_{i j}^{[a \alpha]}-E_{n \mathbf{k}} S_{i j}^{[a \alpha]}+H_{j i}^{*[a \alpha]}\right. \\
& \left.-E_{n \mathbf{k}} S_{j i}^{*[a \alpha]}+V_{\mathrm{i}-\mathrm{e}, i j}^{(a \alpha)}\right] C_{j n \mathbf{k}}+C_{i n \mathbf{k}}^{*}\left[V_{\mathrm{Hxc}, i j}^{\left(\mathscr{E}_{\beta}\right)[a \alpha]}-E_{n \mathbf{k}}^{\left(\mathscr{E}_{\beta}\right)} S_{i j}^{[a \alpha]}\right] C_{j n \mathbf{k}} \\
& +\frac{2 i}{N_{\mathbf{k}}} \sum_{n \mathbf{k}} f_{n \mathbf{k}} \sum_{i} C_{i n \mathbf{k}}^{*}\left\langle\varphi_{i \mathbf{k}}^{(a \alpha)} \mid u_{n \mathbf{k}}^{\left(k_{\alpha}\right)}\right\rangle,
\end{aligned}
$$

где $\varphi_{i \mathbf{k}}^{\prime}$ - периодическая часть $\varphi_{i \mathbf{k}}$, а также использовано обозначение

$$
\{\ldots\}_{i j}^{[a \alpha]}=\left\langle\varphi_{i \mathbf{k}}^{(a \alpha)}|\ldots| \varphi_{j \mathbf{k}}\right\rangle .
$$

Для определения величины полного заряда необходимо учесть ионный вклад, и тогда

$$
Z_{a, \alpha \beta}^{*}=Z_{a}^{(\mathrm{ion})} \delta_{\alpha \beta}+Z_{a, \alpha \beta}^{*(\mathrm{el})},
$$

где $Z_{a}^{(\text {ion })}$ - заряд ионного остова. В заключение отметим, что для базиса, не зависящего от положения атомов, выражение (38) значительно упрощается и переходит в невариационное выражение, полученное в [8] для базиса PW.

\section{5. Результаты и обсуждение}

Схема вычислений отклика на внешнее, однородное электрическое поле, представленная выше, была реализована в настоящей работе с использованием базиса псевдоатомных орбиталей (РАО) [22]. Численные значения РАО находились с помощью решения псевдоатомных уравнений Кона-Шэма для конфигураций основного и частично ионизированных возбужденных состояний с использованием нелокальных, сепарабельных HGH-псевдопотенциалов [23] и далее расщеплялись как DZ-PAO (double- $\xi$ ) для обеспечения большей вариационной гибкости в расчетах электронной структуры кристаллов. Эффекты обмена и электронных корелляций учитывались в локальном приближении по плотности (LDA) $[24,25]$, электронная плотность и ее отклик вычислялись с помощью метода специальных точек [26].

\section{1. $\mathrm{Si}$}

В качестве первого примера рассмотрен кристалл кремния, который является классической системой для проверки расчетных схем и методов. На рис. 1 представлено распределение отклика электронной плотности на внешнее поле, приложенное в направлении [111]. Картина поляризации заряда на связи $\mathrm{Si}-\mathrm{Si}$, а также в окрестности атомов кремния, полученная с использованием базиса локализованных функций, находится в очень хорошем согласии с аналогичным распределением, вычисленным в [27], где эффект экранирования внешнего поля определялся на основе вычисления диэлектрической матрицы.

Результаты вычисления тензора диэлектрической проницаемости представлены на рис. 2, где показана зависимость значений $\varepsilon_{\alpha \beta}^{\infty}$ от плотности $\mathbf{k}$-сетки интегрирования по зоне Бриллюэна вместе с данными, полученными с использованием программы abinit [9] с аналогичными вычислительными параметрами (постоянная решетки,

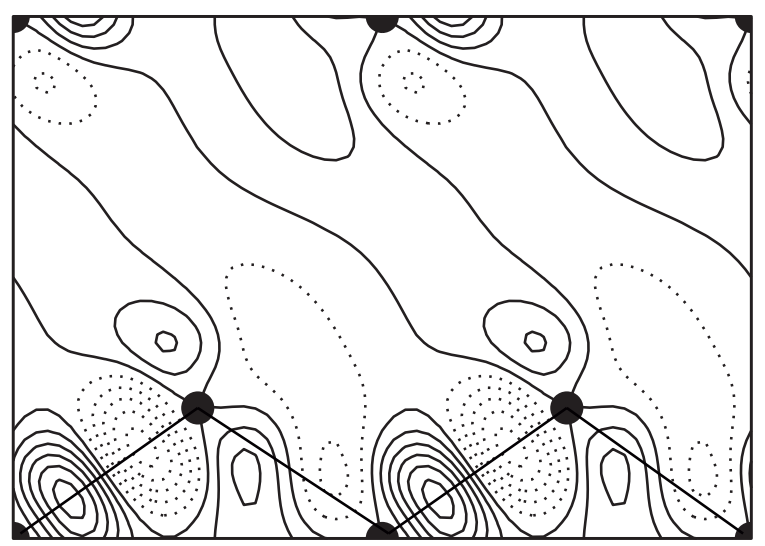

Рис. 1. Отклик электронной плотности $\rho^{(\mathscr{E})}$ в $\mathrm{Si}: \mathscr{E} \uparrow \uparrow 111$; сплошные/пунктирные линии соответствуют положительным/отрицательным значениям $\rho^{(\mathscr{E})}$. 


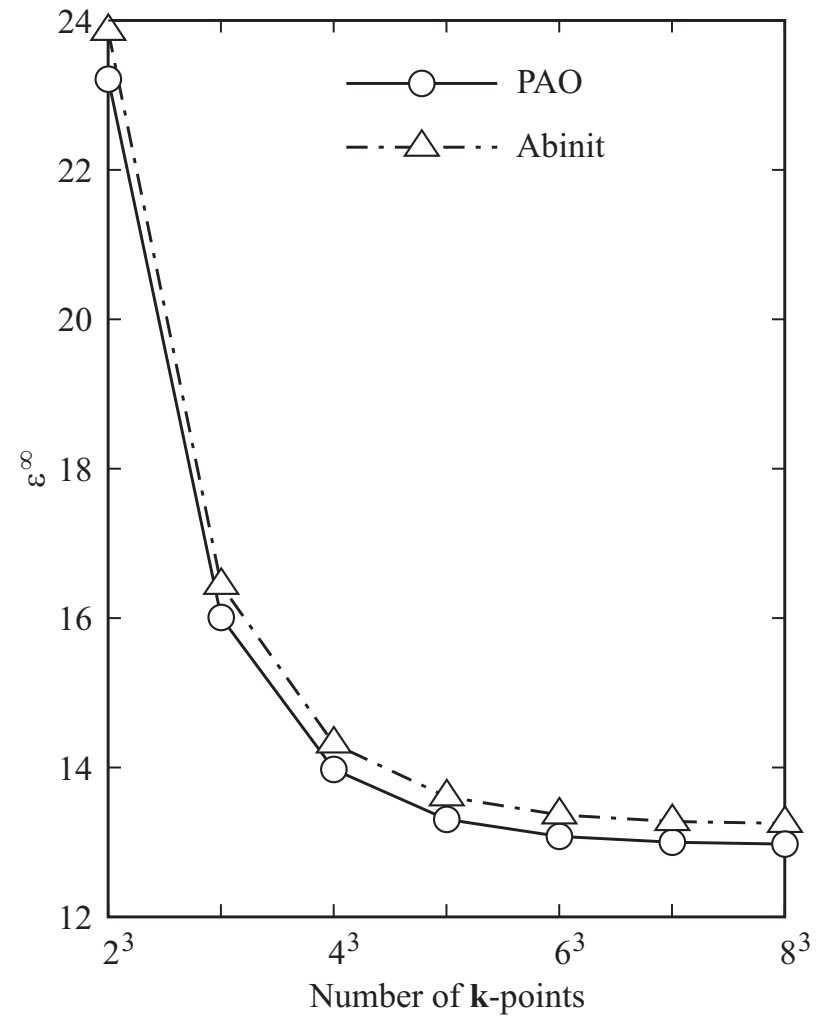

Рис. 2. Сходимость диагональных компонент тензора диэлектрической проницаемости $\mathrm{Si}$ в зависимости от плотности k-сетки.
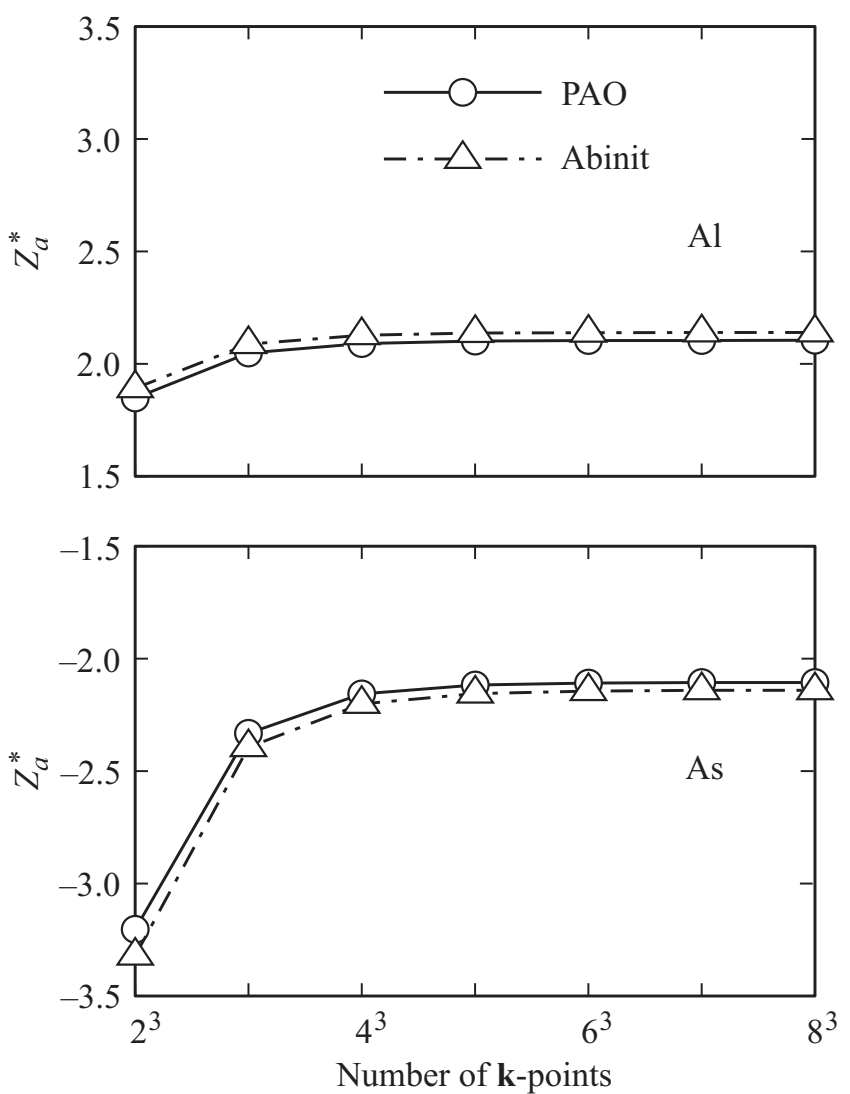

Рис. 3. Сходимость эффективных зарядов AlAs в зависимости от плотности k-сетки (атомные единицы).
HGH-псевдопотенциалы, LDA, энергии обрезания базиca PW). Как видно, результаты находятся в очень хорошем взаимном согласии во всем интервале изменения числа k-точек и для наиболее плотной сетки $(8 \times 8 \times 8)$ составляют соответственно 12.96 (РАО) и 13.23 (PW, abinit).

Отметим, что в обоих случаях расчеты дают для $\varepsilon_{\alpha \beta}^{\infty}$ значение, завышенное в сравнении с экспериментальной величиной 11.7 [28], что уже обусловлено приближенным характером решения многочастичной задачи в рамках DFT.

\subsection{AIAs}

Арсенид алюминия является представителем полупроводниковых соединений типа $\mathrm{A}^{\mathrm{III}} \mathrm{B}^{\mathrm{V}}$ и в данном случае интересен как пробный объект для анализа корректности реализации вычислений динамических зарядов.

В силу симметрии кристалла, а также симметрии атомных положений, тензор эффективных зарядов является изотропным и имеет форму $Z_{a, \alpha \beta}^{*}= \pm Z \delta_{\alpha \beta}$. Как и в случае кристалла кремния, вычисления для AlAs выполнены в двух вариантах — с использованием базисов РАО и $\mathrm{PW}$ (abinit, [9]) для k-сеток с размерностью от $2 \times 2 \times 2$ до $8 \times 8 \times 8$ и общий характер сходимости зарядов представлен на рис. 3. Как видно, во всем интервале изменений плотности $\mathbf{k}$-сеток значения зарядов являются практически идентичными (абсолютная разница порядка 0.03), соответственно, предельные значения составляют \pm 2.10 (PAO) и \pm 2.14 (abinit).

\subsection{LiPN $_{2}$}

Кристалл $\mathrm{LiPN}_{2}$ принадлежит к семейству сильно сжатых халькопиритов, для которых основные параметры кристаллической структуры, такие как тетрагональное сжатие и смещения анинов, заметно отличаются от стандартных для этого класса соединений [29]. Для целей настоящей работы этот кристалл интересен, прежде всего, как пример более сложной (восемь атомов в элементарной ячейке) и низкосимметричной (тетрагональная, объемно-центированная решетка, пространственная группа симметрии $D_{2 d}^{12}$ ) системы с очень заметными различиями в характеристиках связей $\mathrm{Li}-\mathrm{N}$ и $\mathrm{P}-\mathrm{N}$. Расчеты для $\mathrm{LiPN}_{2}$ выполнены с использованием основных структурных и вычислительных параметров, эквивалентных работе [29]; исключение составляют тип псевдопотенциалов и базиса: НGH [23] и РАО вместо ультрамягких псевдопотенциалов [30] и базиса PW [10].

На рис. 4 приведено распределение отклика электронной плотности для поля, приложенного вдоль связей $\mathrm{Li}-\mathrm{N}$ и $\mathrm{N}-\mathrm{P}$ (атомы азота расположены на небольшом удалении от изображенной на рисунках $(a)$ и $(b)$ плоскости, и по этой причине характер распределений $\rho^{(\mathscr{E})}$ для указанных направлений не является абсолютно эквивалентым). Как видно, при наложении поля возникает характерная картина электронной поляризации с 

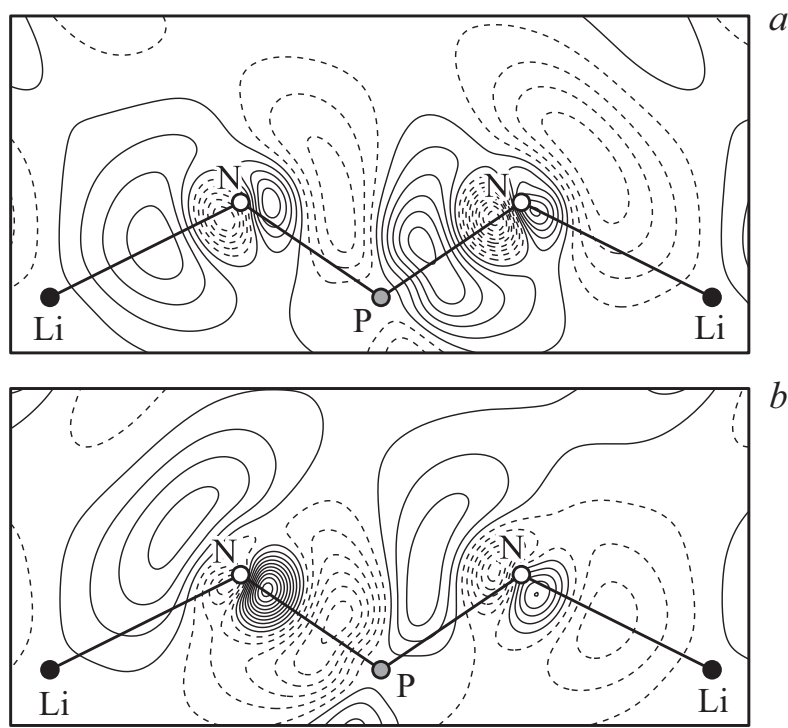

Рис. 4. Отклик электронной плотности $\rho^{(\mathscr{E})}$ в $\mathrm{LiPN}_{2}$ : $\mathscr{E} \uparrow \uparrow \mathrm{Li}-\mathrm{N}(a) ; \mathscr{E} \uparrow \uparrow \mathrm{N}-\mathrm{P}(b)$.

преимущественной локализацией дипольных распределений в окрестности атомов азота, которые в структуре $\mathrm{LiPN}_{2}$, таким образом, играют роль анионов, а заметные отличия в пространственной протяженности $\rho^{(\mathscr{E})}$ вдоль направлений $\mathrm{Li}-\mathrm{N}$ и $\mathrm{N}-\mathrm{P}$ указывают, в свою очередь, на различный характер химической связи для указанных пар атомов.

Качественные выводы, приведенные выше, находятся в полном согласии с аналогичными выводами [29], полученными на основе анализа эффективных зарядов

Таблица 1. Значения компонент тензора диэлектрической проницаемости $\varepsilon_{\alpha \beta}^{\infty}$ для кристалла $\mathrm{LiPN}_{2}$ вдоль оси $z\left(\varepsilon_{\|}^{\infty}\right)$ и перпендикулярно ей $\left(\varepsilon_{\perp}^{\infty}\right)$

\begin{tabular}{c|c|c}
\hline Компонента & Базис РАО & Базис PW, [29] \\
\hline$\varepsilon_{\|}^{\infty}$ & 4.37 & 4.43 \\
$\varepsilon_{\perp}^{\infty}$ & 4.17 & 4.18
\end{tabular}

Таблица 2. Значения эффективных зарядов Борна $Z_{a, \alpha \beta}^{*}$ для кристалла $\mathrm{LiPN}_{2}$ (атомные единицы)

\begin{tabular}{c|c|c}
\hline Атом & Базис РАО & Базис $\mathrm{PW},[29]$ \\
\hline$Z_{\mathrm{Li}, \alpha \beta}^{*}$ & $\left(\begin{array}{rrr}1.06 & 0.05 & 0.00 \\
-0.05 & 1.06 & 0.00 \\
0.00 & 0.00 & 1.01\end{array}\right)$ & $\left(\begin{array}{rrr}1.02 & 0.05 & 0.00 \\
-0.05 & 1.02 & 0.00 \\
0.00 & 0.00 & 1.03\end{array}\right)$ \\
\hline \multirow{2}{*}{$Z_{\mathrm{P}, \alpha \beta}^{*}$} & $\left(\begin{array}{rrr}3.79 & -0.83 & 0.00 \\
0.83 & 3.79 & 0.00 \\
0.00 & 0.00 & 4.00\end{array}\right)$ & $\left(\begin{array}{rrr}3.78 & -0.83 & 0.00 \\
0.83 & 3.78 & 0.00 \\
0.00 & 0.00 & 4.03\end{array}\right)$ \\
\hline \multirow{2}{*}{$Z_{\mathrm{N} 1, \alpha \beta}^{*}$} & $\left(\begin{array}{rrr}-1.94 & 0.00 & 0.00 \\
0.00 & -2.91 & -0.95 \\
0.00 & -0.97 & -2.51\end{array}\right)$ & $\left(\begin{array}{rrr}-1.91 & 0.00 & 0.00 \\
0.00 & -2.92 & -0.97 \\
0.00 & -0.98 & -2.54\end{array}\right)$
\end{tabular}

в $\mathrm{LiPN}_{2}$, которые, включая также тензор диэлектрической проницаемости, приведены в табл. 1,2 вместе с результатами настоящей работы. Как видно, значения $Z_{a, \alpha \beta}^{*}$ и $\varepsilon_{\alpha \beta}^{\infty}$ находятся в очень хорошем количественном согласии с результатами [29], различия лежат в пределах нескольких процентов, что, как и ранее, следует связать с использованием базиса локализованных функций.

\section{6. Заключение}

В настоящей работе в рамках теории возмущений для функционала плотности сформулирована схема расчета отклика кристаллов на внешнее, однородное электрическое поле с использованием базиса локализованных функций в форме псевдоатомных орбиталей. Расчеты, выполненные для различных по типу соединений, показывают общую корректность реализованного подхода, а также достаточную гибкость базиса PAО для количественных оценок важных характеристик кристаллических систем.

\section{Список литературы}

[1] P. Hohenberg, W. Kohn. Phys. Rev. 136, B864 (1964).

[2] W. Kohn, L.J. Sham. Phys. Rev. 140, A1133 (1965).

[3] W.E. Pickett. Comput. Phys. Rep. 9, 115 (1989).

[4] M.C. Payne, M.P. Teter, D.C. Allan, T.A. Arias, J.D. Joannopoulos. Rev. Mod. Phys. 64, 1045 (1992).

[5] Н.Е. Зейн. ФТТ 26, 3024 (1984).

[6] S. Baroni, S. de Gironcoli, A. Dal Corso, P. Giannozzi. Rev. Mod. Phys. 73, 515 (2001).

[7] X. Gonze. Phys. Rev. B 55, 10337 (1997).

[8] X. Gonze, C. Lee. Phys. Rev. B 55, 10355 (1997).

[9] X. Gonze, B. Amadon, P.-M. Anglade, J.-M. Beuken, F. Bottin, P. Boulanger, F. Bruneval, D. Caliste, R. Caracas, M. Cote, T. Deutsch, L. Genovese, P. Ghosez, M. Giantomassi, S. Goedecker, D. Hamann, P. Hermet, F. Jollet, G. Jomard, S. Leroux, M. Mancini, S. Mazevet, M. Oliveira, G. Onida, Y. Pouillon, T. Rangel, G.-M. Rignanese, D. Sangalli, R. Shaltaf, M. Torrent, M. Verstraete, G. Zerah, J. Zwanziger. Comput. Phys. Commun. 180, 2582 (2009).

[10] P. Giannozzi, S. Baroni, N. Bonini, M. Calandra, R. Car, C. Cavazzoni, D. Ceresoli, G.L. Chiarotti, M. Cococcioni, I. Dabo, A.D. Corso, S. de Gironcoli, S. Fabris, G. Fratesi, R. Gebauer, U. Gerstmann, C. Gougoussis, A. Kokalj, M. Lazzeri, L. Martin-Samos, N. Marzari, F. Mauri, R. Mazzarello, S. Paolini, A. Pasquarello, L. Paulatto, C. Sbraccia, S. Scandolo, G. Sclauzero, A.P. Seitsonen, A. Smogunov, P. Umari, R.M. Wentzcovitch. J. Phys.: Condens. Matter 21, 395502 (2009).

[11] G.B. Bachelet, D.R. Hamann, M. Schluter. Phys. Rev. B 26, 4199 (1982).

[12] P. Pulay. Mol. Phys. 17, 197 (1969).

[13] H. Hellmann. Einfuhrung in die Quantenchemie. Deuticke, Leipzig (1937). P. 61, 285.

[14] R.P. Feynman. Phys. Rev. 56, 340 (1939).

[15] S. Baroni, P. Giannozzi, A. Testa. Phys. Rev. Lett. 58, 1861 (1987).

[16] R. Yu, H. Krakauer. Phys. Rev. B 49, 4467 (1994). 
[17] S.Y. Savrasov. Phys. Rev. B 54, 16470 (1996).

[18] R. Heid, K.-P. Bohnen. Phys. Rev. B 60, R3709 (1999).

[19] R. Kouba, A. Taga, C. Ambrosch-Draxl, L. Nordstrom, B. Johansson. Phys. Rev. B 64, 184306 (2001).

[20] H. Shang, C. Carbogno, P. Rinke, M. Scheffler. Comput. Phys. Commun. 215, 26 (2017).

[21] J.A. Pople, R. Krishnan, H.B. Schlegel, J.S. Binkley. Int. J. Quantum Chem. 16, 225 (1979).

[22] A.B. Gordienko, A.S. Poplavnoi. Phys. Status Solidi B 202, 941 (1997).

[23] C. Hartwigsen, S. Goedecker, J. Hutter. Phys. Rev. B 58, 3641 (1998).

[24] J.P. Perdew, A. Zunger. Phys. Rev. B 23, 5048 (1981).

[25] D.M. Ceperley, B.J. Alder. Phys. Rev. Lett. 45, 566 (1980).

[26] H.J. Monkhorst, J.D. Pack. Phys. Rev. B 13, 5188 (1976).

[27] A. Baldereschi, R. Car, E. Tosatti. Solid State Commun. 32, 757 (1979)

[28] M.S. Hybertsen, S.G. Louie. Phys. Rev. B 35, 5585 (1987).

[29] A.V. Kosobutsky. J. Phys.: Condens. Matter 21, 405404 (2009).

[30] D. Vanderbilt. Phys. Rev. B 41, 7892 (1990).

Редактор Е.Ю. Флегонтова 